\title{
Confronto tra sutura con antibatterico vs sutura standard nella prevenzione delle infezioni del sito chirurgico
}

\author{
Comparison between antibacterial coated suture vs standard suture to prevent \\ the surgical site infections
}

\author{
Elisabetta Palma 1 \\ Nicoletta Brocanelli2 \\ Antonella Luconi3 \\ Giancarlo Cicolini4
}

RIASSUNTO

Le infezioni del sito chirurgico rappresentano ancora oggi un problema aperto, con prolungamento dei tempi di degenza e relativo aumento dei costi sanitari ed assicurativi. La recente letteratura evidenzia come il triclosan ${ }^{\circledR}$ possa essere utilizzato con sicurezza per ridurre l'incidenza di infezioni. In questo studio vengono confrontati due gruppi di pazienti sottoposti in regime di elezione a chirurgia "pulita". Il follow up, condotto trenta giorni dopo l'intervento chirurgico attraverso intervista telefonica, come da protocollo del Sistema Nazionale di sorveglianza delle infezioni del sito chirurgico, ha mostrato migliori outcomes, anche se non statisticamente significativi, in termini di prevenzione del sito chirurgico, utilizzando le suture con antibatterico rispetto a quelle standard.

Parole chiave: infezioni del sito chirurgico, sicurezza, gestione del rischio

\section{ABSTRACT}

The surgical site infections (SSI) still represent an issue, which causes prolonged hospital staying, major costs for both healthcare system and professional insurance. Recent studies enlighten that using the antibacterial agent triclosan ${ }^{\circledR}$ leads to decrease infections rates. In this observational study we compared two groups of patients undergoing scheduled the so called clean surgery. The absence of SSI was assessed in all patients, 30 days later the surgery, through a telephone interview as indicated by the National Group for SSI surveillance protocol. The use of antibacterial sutures shows to be successful in preventing the SSI in comparison to standard sutures, even if results are not statistically significant.

Keywords: surgical site infections, safety management, risk management

\section{INTRODUZIONE}

I termine "infezioni del sito chirurgico" (SSI) defi1 nisce quelle infezioni che insorgono entro 30 giorni dall'atto operatorio (ma tale periodo sale ad un anno in caso di impianti) e che interessano l'incisione o $\mathrm{i}$ tessuti profondi (Owens \& Stoessel, 2008). Le SSI, classificate da tempo dal Centers for Disease Control and Prevention (CDC) di Atlanta, USA (Horan et al. 1992), determinano un serio problema clinico, rappresentando la seconda più comune infezione correlata all'assistenza (ICA) tra i pazienti ricoverati. Esistono sistemi di sorveglianza su una rete di ospedali sentinella a livello nazionale, i più noti sono il National Nosocomial Infections Surveillance System (NNIS) statuni-

1 Dottore di ricerca, Università G. d'Annunzio Chieti-Pescara

2 Coordinatore BOC ASUR Marche Area Vasta 2 Senigallia

3 Coordinatore Infermieristico Dipartimentale, ASUR Marche Area Vasta 2 Jesi

4 Dottore di Ricerca, Direttore Didattico CDLM Scienze Infermieristiche e Ostetriche, Università G. d'Annunzio Chieti-Pescara g.cicolini@unich.it tense e il Progetto europeo Hospitals in Europe Link for Infection Control through Surveillance (HELICS) a cui però l'Italia non partecipa. Sono stati tuttavia condotti numerosi studi multicentrici di prevalenza che permettono di stimare che nel nostro Paese si verificano 450-700 mila infezioni ospedaliere (5-8\% dei pazienti ricoverati), responsabili di 1350-2100 decessi prevenibili ogni anno (Istituto Superiore di Sanità, 2011).

Vari studi indicano tassi di incidenza che oscillano tra il 6,3\% e il $14,8 \%$ (Delgado et al. 2001; Reilly et al. 2006), fino al 20\% (Owens \& Stoessel, 2008).

Il principale fattore di rischio per l'infezione del sito chirurgico è rappresentato dalla tipologia di intervento. In tal senso, la classica suddivisione della ferita chirurgica in base al livello di contaminazione (pulita, pulitacontaminata, contaminata e sporca) resta la guida fondamentale nella valutazione del rischio. Pur considerando i limiti associati alla differente conduzione delle procedure di sorveglianza post-dimissione, i tassi di SSI riportati in letteratura in funzione della tipologia di ferita corrispondono a: 
- $<2 \%$ per le ferite chirurgiche pulite,

- al $2 \%$ per quelle pulite-contaminate,

- al 3\% per le ferite contaminate,

- all'8\% per quelle sporche.

Altri fattori in gioco per la valutazione del rischio (Gaynes et al, 2001) sono rappresentati dalla durata dell'intervento, dal tipo di approccio (laparotomico, laparoscopico) e dalla classe anestesiologica ASA (Owens et al. 1978). Oltre a ciò, vanno considerati i fattori di rischio legati al paziente, come l'obesità (Anaya \& Dellinger, 2006) e il diabete mellito scompensato (Dellinger, 2001) mentre, il ruolo del fumo di tabacco è ancora controverso (Delgado et al. 2003).

Negli USA si stima che ci siano mediamente 500.000 nuove infezioni l'anno su un totale di circa 27 milioni di procedure, pari a un quarto di tutte le infezioni ospedaliere (CDC, 1994; Mangram et al. 1999; Nichols et al. 2001).

I dati italiani riportano un'incidenza delle SSI paragonabile a quella europea (Moro et al. 2004; Florio et al. 2006; Petrosillo et al. 2008). Uno studio del Gruppo Italiano per lo Studio delle Infezioni Nosocomiali per il biennio 2002-2004 stima nel nostro Paese una prevalenza delle infezioni associate all'assistenza (Healthcare Associated Infections - HAIs) del 6.7\%, di cui il $12.2 \%$ è rappresentato dalle infezioni del sito chirurgico (Lanini et al. 2009).

Le SSI incidono ancora oggi sulla prognosi dei pazienti in termini di morbosità, mortalità ma anche di allungamento dei tempi medi di degenza e dei costi ospedalieri (Kirkland et al, 1999; Monge Jodra V et al, 2006; Olsen et al, 2008).

Le linee guida per la Prevenzione delle Infezioni della Ferita Chirurgica (CDC, 1999) forniscono raccomandazioni finalizzate al contenimento del rischio. Ogni raccomandazione è classificata sulla base di dati scientifici esistenti, razionali teorici ed applicabilità.

In generale, la prevenzione delle SSI dovrebbe prevedere una serie combinata di interventi, che includono la tricotomia, la somministrazione della profilassi antibiotica, il controllo della temperatura corporea in fase peri-operatoria e il controllo della glicemia, in particolare nei pazienti diabetici. Ulteriori dispositivi utili per il controllo delle SSI includono i bendaggi e le suture con antibatterico, che consentono la creazione di una zona di inibizione della crescita batterica).

I dispositivi medici non causano infezioni, tuttavia, è noto che contribuiscono al potenziamento delle infezioni stesse. Una volta che un dispositivo medico è colonizzato, i batteri sono meno suscettibili al trattamento antibiotico, a causa della formazione del biofilm. In queste condizioni, il numero di microrganismi in grado di trasmettere le infezioni è più basso ed il dispositivo impiantato funziona come veicolo per la diffusione del batterio ai tessuti circostanti. Le suture con antibatterico sono inerti, non antigenetiche, apirogene e sollecitano una blanda reazione tissutale durante l'assorbimento, e vari studi hanno già confrontato queste suture e studiato la loro utilizzabilità (Storch et al, 2002; Ford et al, 2005) oltre che la loro sicurezza di utilizzo (Leaper et al. 2011; Galal \& El-Hindawy, 2011). Uno studio multicentrico randomizzato (Huszàr et al., 2012) su 485 pazienti sottoposti a chirurgia addominale maggiore non ha evidenziato differenze statisticamente significative nel tasso di SSI tra il gruppo in cui è stata utilizzata la sutura con triclosan e quello in cui è stata usata la sutura standard. Una revisione sistematica con metanalisi di tutti i trial clinici randomizzati e controllati riguardanti l'uso di suture con Triclosan ha evidenziato come la sutura con antibatterico non riduca in modo statisticamente significativo il tasso di SSI e suggerisce ulteriori studi prima di consigliarne l'uso clinico di routine (Chang et al., 2012).

La sutura Vicryl PLUSTM è una sutura sintetica assorbibile intrecciata, composta da Polyglactin 910, un copolimero di lattide (acido lattico al 10\%) e di glicolide (acido glicolico al 90\%). Il rivestimento è composto in parti uguali da Polyglactin 370 e da stearato di calcio, con proprietà lubrificante, aderente alla sutura, con il vantaggio che la sutura viene poi assorbita rapidamente, finita la funzione meccanica di supporto. A questi elementi è stato aggiunto IRGACARE MP®, la forma più pura di Triclosan, un agente antibatterico ad ampio spettro che crea una zona di inibizione alla diffusione batterica svolgendo un'azione preventiva nei confronti delle possibili infezioni del sito chirurgico.

Tra i fattori che più comunemente contribuiscono alle infezioni si annoverano il profilo del paziente, la tecnica chirurgica, la situazione ambientale operatoria e post-operatoria (sepsi), la tipologia di intervento (impianti).

La sutura con antibatterico neutralizza una di queste variabili: la sutura, come impianto, non è più un fattore di contribuzione alle infezioni del sito chirurgico, e una precauzione in più per minimizzare il rischio che il paziente contragga un'infezione della ferita chirurgica.

Scopo del presente lavoro è quello di verificare se l'utilizzo delle suture antibatteriche è in grado di ridurre il numero delle SSI versus suture senza triclosan ${ }^{\circledR}$ in pazienti sottoposti a chirurgia pulita d'elezione.

\section{METODO}

Studio osservazionale retrospettivo. Dalla documentazione sanitaria (schede pazienti e schede di followup) relative a 400 interventi chirurgici sono stati estra- 
polati casualmente (tramite generatore di numeri random) i dati di 132 pazienti per ottenere una potenza dell' $80 \%$. Sono stati presi in esame gli interventi chirurgici effettuati presso l' ASUR Marche Area Vasta 2 (70 a Senigallia e 62 a Jesi, AN).

Nel presidio di Senigallia viene utilizzato di routine il filo con antibatterico, sia monofilamento (MONOCRYL PLUSTM) che intrecciato (VICRYL PLUSTM), mentre a Jesi vengono utilizzati i fili omologhi ma senza antibatterico (MONOCRYL ${ }^{\mathrm{TM}}$ e VICRYL ${ }^{\mathrm{TM}}$ ).

\section{Criteri di inclusione}

Sono stati inclusi nello studio tutti gli interventi di elezione (programmati) per le seguenti indicazioni e patologie:

- ernia inguinale monolaterale (DRG 55090),

- calcolosi della colecisti (DRG 57420),

- nodulo mammario (DRG 217),

- tumore della mammella (DRG 1744),

- noduli tiroidei (DRG 2411).

\section{Criteri di esclusione}

Sono stati esclusi dallo studio i pazienti sottoposti a intervento in regime di urgenza ed emergenza, sottoposti a interventi c.d. "sporchi" o contaminati (es. tumori del colon retto), pazienti affetti da patologie (es. diabete mellito, peritoniti) o altre comorbilità che elevano il rischio di infezioni (classificate dagli anestesisti come ASA3 e ASA4).

La raccolta dei dati dei pazienti per il follow up a 30 giorni è stata effettuata con intervista telefonica guidata, con le modalità previste dal protocollo del Sistema Nazionale di sorveglianza delle infezioni del sito chirurgico (Ccm, 2009).

E'stata richiesta l'autorizzazione allo studio alle rispettive Direzioni Mediche di Presidio.

\section{RISULTATI}

\section{Verifica dell'omogeneità dei gruppi di pazienti}

I pazienti rispondenti ai criteri di inclusione sono stati analizzati per verificare la loro omogeneità rispetto ad alcune variabili demografiche (età e genere) e al fumo di sigaretta (Tab. 1).

\begin{tabular}{|c|c|c|c|c|c|c|}
\hline & pazienti $n$ & $\begin{array}{c}\text { età media } \\
\text { (DS) }\end{array}$ & U & D & fumatori & $p$ \\
\hline Senigallia & 70 & $\begin{array}{c}55.91 \\
(15.06)\end{array}$ & 40 & 30 & 26 & $p=0.42$ \\
\hline Jesi & 62 & $\begin{array}{c}58.14 \\
(16.72)\end{array}$ & 33 & 29 & 30 & \\
\hline
\end{tabular}

Tabella 1 - confronto dei pazienti con test ANOVA
Il test ANOVA utilizzato per confrontare i due gruppi di pazienti non ha rilevato differenze significative $(\mathrm{p}=0,42)$

Relativamente alla variabile fumo di sigaretta e infezione della ferita, abbiamo notato che nei 4 casi di Senigallia e nei 4 di Jesi la proporzione di fumatori e non fumatori era equamente distribuita.

Anche relativamente alla presenza di comorbilità, i due gruppi risultano molto simili, in quanto la distribuzione dei pazienti in base alla classificazione ASA è quasi sovrapponibile (Tab.2) e il test t di Student per campioni indipendenti restituisce un valore non significativo $(\mathrm{p}=0,87)$

\begin{tabular}{|c|c|c|c|}
\hline & ASA1 & ASA2 & $p$ \\
\hline Senigallia & 52 & 18 & \multirow{2}{*}{$p=0.87$} \\
\cline { 1 - 3 } Jesi & 46 & 16 & \\
\hline
\end{tabular}

Tabella 2 - distribuzione in base alla classificazione ASA

\section{Analisi dei dati relativi agli interventi}

Relativamente al motivo dell'intervento, si evidenzia (Fig.1) come la maggiore proporzione di interventi effettuati a Senigallia è rappresentato dall'ernioplastica (DRG 55090), mentre a Jesi l'intervento più praticato è la colecistectomia (DRG 57420).

L'analisi delle distribuzioni della variabile "tipologia di intervento chirurgico" nei due gruppi con il test t di Student per campioni non appaiati non appare statisticamente significativa $(p=0,76)$, permettendoci di affermare che i due gruppi non differiscono in maniera significativa per la variabile DRG in oggetto.

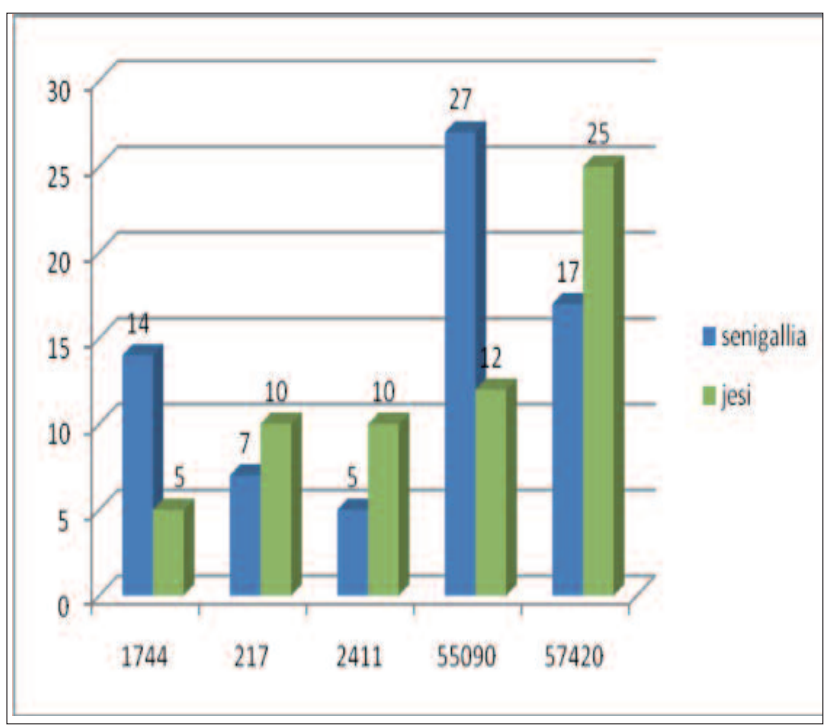

Figura 1- distribuzione per DRG

A tutti i pazienti operati è stato effettuato il followup a 30 giorni per verificare 1 avvenuta guarigione della ferita chirurgica. Tale dato è stato raccolto attraverso una intervista telefonica. 


\begin{tabular}{|c|c|c|c|c|c|c|c|}
\hline & DRG & 1744 & 217 & 2411 & 55090 & 57420 & totali \\
\hline Senigallia & SSI & 14 & 7 & 5 & 27 & 17 & 70 \\
\hline (sutura con triclosan ${ }^{-}$) & si & 0 & 1 & 0 & 1 & 2 & 4 \\
\hline Jesi & no & 5 & 10 & 10 & 12 & 25 & 62 \\
\hline (sutura standard) & si & 1 & 0 & 0 & 1 & 2 & 4 \\
\hline
\end{tabular}

Tabella 3 - distribuzione per DRG e frequenza di ferite infette

La Tab. 3 evidenzia il dato delle ferite non guarite a 30 giorni (quindi considerate infette) suddivise in base al DRG che ha determinato il tipo di intervento chirurgico.

I dati raccolti attraverso l'intervista telefonica hanno evidenziato la presenza di 8 soggetti con SSi su un totale di 132 pazienti, equamente distribuiti tra i due gruppi.Tale dato non appare statisticamente significativo (test esatto di Fischer, p value $=0,57$ ), tuttavia la stima del rischio (SW open source Openepi, http://www.openepi.com) evidenzia un RR pari a 0,8 (IC 95\% 0,23-3,39) e un OR pari a 0,87 (IC95\% 0,21 - 3,67). La frazione prevenibile nei pazienti globalmente considerati è pari al 6\% (IC 95\% -186$43,8)$ e l'NNH (number needed to harm) pari a 136 (IC 95\% $11-\infty$ ).

\section{DISCUSSIONE}

In letteratura è stata già valutata l'efficacia del triclosan per la prevenzione delle SSI. Alcuni autori hanno riportato risultati positivi in interventi puliti di elezione a carico della mammella (Laas et al, 2012), mentre altri non hanno notato differenze significative nei assi di SSI (Williams et al, 2013). Sembra che benefici potrebbero essere attesi persino nella chirurgia addominale (Heger et al, 2011).

La letteratura secondaria non fornisce ulteriori prove di efficacia: la revisione sistematica di Chang et al (2012) non ha evidenziato differenze rilevanti tra le suture standard e quelle con antibatterico, mentre quella di Wang et al (2013) si.

Lo studio ha preso in esame i dati di 132 pazienti tra tutti quelli rispondenti ai criteri di elezione e sottoposti a interventi "puliti" in regime programmato.

I due gruppi di pazienti sono risultati simili per quanto riguarda le variabili demografiche (età), l'abitudine tabagica e per la variabile DRG.

I tassi di SSI non differiscono in modo statisticamente significativo tra $\mathrm{i}$ due gruppi. Il gruppo di pazienti su cui è stato utilizzato il filo di sutura con antibatterico ha evidenziato tuttavia un rischio di SSI pari a 0,05 ; di poco inferiore a quello del gruppo operato con filo senza antibatterico $(0,06)$. I dati evidenziano una associazione protettiva, anche se debole, tra l'uso di sutura con antibatterico e il rischio di SSI.

\section{LIMITI DELLO STUDIO}

A fronte dei circa 3000 interventi chirurgici/anno che rappresentano la casistica totale a disposizione, si è scelto di limitare il numero di pazienti eleggibili per la necessità di eliminare tutti i possibili bias che potevano interferire con la variabile di studio, ovvero la presenza di comorbilità, di rischi legati al tipo di intervento (es. interventi sporchi) o alla sua tempistica (interventi in emergenza o urgenza).

\section{CONCLUSIONI}

I fili di sutura utilizzati nelle due realtà messe a confronto appartengono alle stesse case produttrici e a parte la presenza di antibatterico - sono perfettamente confrontabili circa le loro caratteristiche tecniche. La sicurezza nell'uso delle suture con triclosan ${ }^{\circledR}$ è stata già verificata in letteratura su diversi scenari operatori, attraverso valutazioni sia delle caratteristiche tecniche che del rischio di sviluppo di resistenze da parte dei microbi.

Circa le valutazioni economiche non si evidenziano maggiori costi per l'acquisto delle suture con antibatterico, in quanto a livello regionale la casa produttrice ha concordato l'equivalenza dei prezzi tra i fili di sutura con triclosan ${ }^{\circledR}$ rispetto alle suture tradizionali.

Raccomandazioni per la pratica clinica: la letteratura secondaria più recente sull'argomento non ha evidenziato riduzioni statisticamente significative nei tassi di incidenza delle SSI correlate all'uso di suture con antibatterico. Il rischio di SSI deriva da un complesso mix di variabili, solo alcune delle quali legate al paziente. Relativamente allo studio in esame, esso ha interessato solo pazienti a basso rischio di SSI, classificati come ASA 1 e 2 , che si sono sottoposti ad intervento chirurgico in elezione.

In questo gruppo di soggetti, l'utilizzo della sutura con antibatterico sembra avere un effetto protettivo 
debole nei pazienti, dunque può essere raccomandato utilizzare questo tipo di suture, data anche l'assenza di maggiori costi legati alla fornitura. L'uso di tali presidi non può in nessun caso sostituire la corretta prassi come evidenziato dalle raccomandazioni per la prevenzione delle SSI.

\section{BIBLIOGRAFIA}

Agenzia Regionale della Sanità, Regione Friuli Venezia Giulia, Ferita chirurgica (2002). Report Regionale 2000/2001. Data accesso 16 ottobre 2010, da: http://www.snlg-iss.it/cms/files/PNLG_antib_impa.pdf

Anaya, D.A.\& Dellinger, E.P. (2006) The obese surgical patient: a susceptible host for infection. Surgical Infections (Larchmt) 7(5): 473-80.

Centers for Disease Control and Prevention, National Center for Health Statistics Vital and Health Statistics, Detailed diagnoses and procedures national hospital discharge survey 1994 (1997). Vol 12 7. Hyattsville (MD): Department of Health and Human Services, 1997.

Centro nazionale per la prevenzione e il Controllo delle Malattie (Ccm). Sorveglianza, prevenzione e controllo. Infezioni correlate all'assistenza. Sicurezza del paziente: il rischio infettivo (2008-2010). Sistema Nazionale Sorveglianza Delle Infezioni Del Sito Chirurgico Protocollo. ASSR Emilia-Romagna, Bologna, novembre 2009. Data accesso 6 gennaio 2012, da: asr.regione.emilia-romagna.it/.../CCM_Protocollo _ISC_11_09.pdf Simili

Chang, W.K., Srinivasa, S., Morton, R. \& Hill A.G. (2012) Triclosan-impregnated sutures to decrease surgical site infections: systematic review and meta-analysis of randomized trials. Annals of Surgery, 255(5):854-9.

Delgado-Rodríguez , M., Gómez-Ortega, A., Sillero-Arenas, M. \& Llorca, J. (2001) Epidemiology of surgical-site infections diagnosed after hospital discharge: a prospective cohort study. Infection Control and Hospital Epidemiology, 22(1): 24-30.

Delgado-Rodriguez, M., Medina-Cuadros, M., MartínezGallego, G, Gómez-Ortega, A., Mariscal-Ortiz, M., Palma-Pérez, S. \& Sillero-Arenas, M. (2003) A prospective study of tobacco smoking as a predictor of complications in general surgery. Infection Control and Hospital Epidemiology, 24(1): 37-43.

Dellinger, E.P. (2001) Preventing surgical-site infections: the importance of timing and glucose control. Infection Control and Hospital Epidemiology, 22(10): 604-6.

Edmiston Jr, C.E., Candace, J., Krepel, P., Wilson, J., Grahn, F.B., Sadenwasser, P.J., Welter, D.L. \& Seabrook, G.R. (2008) Reducing the risk of surgical site infections: embracing basic and innovative risk reduction strategies, Healthcare Infection, 13(4) -121

Fiorio, M., Marvaso, A., Viganò, F. \& Marchetti, F. (2006) Incidence of surgical site infections in general surgery in Italy. Infection 2006; 34(6): 310-4.

Ford, H.R., Jones, P., Gaines, B., Reblock, K. \& Simpkins, D.L. (2005) Intraoperative Handling and Wound
Healing: Controlled Clinical Trial Comparing Coated VICRYL ${ }^{\circledR}$ Plus Antibacterial Suture (Coated Polyglactin 910 Suture with Triclosan) with Coated VICRYL® Suture (Coated Polyglactin 910 Suture), Surgical Infections, 6(3):313-321

Galal, I. \& El-Hindawy, K. (2011) Impact of using triclosanantibacterial sutures on incidence of surgical site infection. American Journal of Surgery, 202(2):133-8. Epub 2011 May 19.

Gaynes, R.P, Culver, D.H., Horan, T.C., Edwards, J.R., Richards, C. \& Tolson, J.S.(2001) Surgical site infection (SSI) rates in the United States, 1992-1998: the National Nosocomial Infections Surveillance System basic SSI risk index. Clinical Infectious Disease 1; 33 (Suppl 2): S69-77.

Heger, U., Voss, S., Knebel, P., Doerr-Harim, C., Neudecker, J., Schuhmacher, C., Faist, E., Diener, M.K., Kieser, M. \& Seiler, C.M. (2011) Prevention of abdominal wound infection (PROUD trial, DRKS00000390): study protocol for a randomized controlled trial. Trials, 21, 12:245.

Horan, T.C., Gaynes, R.P., Martone, W.J., Jarvis, W.R. \& Emori, T.G. (1992) CDC definitions of nosocomial surgical site infections 1992: a modification of CDC definitions of surgical wound infections. Infection Control and Hospital Epidemiology, 13(10): 606-8.

Huszár, O., Baracs, J., Tóth, M., Damjanovich, L., Kotán, R., Lázár, G., Mán, E., Baradnai, G., Oláh, A., Benedek-Tóth, Z., Bogdán-Rajcs, S., Zemanek, P., Oláh, T.,Somodi, K., Svébis, M., Molnár, T. \& Horváth, Ö.P. (2012) Comparison of wound infection rates after colon and rectal surgeries using triclosan-coated or baresutures -- a multi-center, randomized clinical study. Magyar Sebészet Jun;65(3):83-91.

Improving patient safety in Europe Data accesso 6 gennaio 2012, da: http://helics.univ-lyon 1.fr/documents/ documents.htm

Istituto Superiore di Sanità - Centro Nazionale di Epidemiologia, Sorveglianza e Promozione della Salute (2011). Infezioni correlate all'assistenza, aspetti epidemiologici. Aggiornato in data martedì 6 settembre 2011. Data accesso 5 novembre 2012, da: http://www.epicentro.iss.it/problemi/infezioni_correlat e/epid.asp

Kirkland, K.B., Briggs, J.P., Trivette, S.L., Wilkinson, W.E. \& Sexton, D.J. (1999) The impact of surgical-site infections in the 1990s: attributable mortality, excess length of hospitalization, and extra costs. Infection Control and Hospital Epidemiology;20(11): 725-30.

Lanini, S., Jarvis, W.R., Nicastri, E., Privitera, G., Gesu, G., Marchetti, F., Giuliani, L., Piselli, P., Puro, V., Nisii, C., Ippolito, G. \& INF-NOS Study Group (Gruppo Italiano per lo Studio delle Infezioni Noscomiali) (2009) Healthcare-associated infection in Italy: annual pointprevalence surveys, 2002-2004. Infection Control and Hospital Epidemiology, 30(7):659-65.

Laas, E., Poilroux, C., Bézu, C., Coutant, C., Uzan, S., Rouzier, R. \& Chéreau, E. (2012) Antibacterial-coated suture in reducing surgical site infection in breast surgery: a 
prospective study. International Journal of Breast Cancer: 819578.

Leaper, D. (2010) Risk Factors for and Epidemiology of Surgical Site Infections, Surgical Infections, 11(3): 283-287

Leaper, D., Assadian, O., Hubner, N.O., McBain, A., Barbolt, T., Rothenburger, S. \& Wilson, P. (2011) Antimicrobial sutures and prevention of surgical site infection: assessment of the safety of the antiseptic triclosan. International Wound Journal, 8(6):556-66.

Mangram, A. J., Horan, T. C. \& Pearson, M. L. (1999) CDC Guideline for prevention of surgical site infection. Infections Control and Hospital Epidemiology, 20(4), 247278

Martinelli, D. \& Germinario, C. (2009) Epidemiologia delle infezioni del sito chirurgico, Annali di Igiene, 21 (Suppl. 1): 3-7

Monge Jodra, V, Sainz de Los Terreros Soler, L., Diaz-Agero Perez, C., Saa Requejo, C.M. \& Plana Farras, N. (2006) Excess length of stay attributable to surgical site infection following hip replacement: a nested casecontrol study. Infections Control and Hospital Epidemiology; 27(12): 1299-303.

Moro, M.L., Petrosillo, N. \& Siepi, G. (2001) Indagine conoscitiva nazionale sulle attività di sorveglianza e controllo delle infezioni ospedaliere negli ospedali pubblici italiani. Rapporti ISTISAN. Data accesso 15 gennaio 2011, da: http://asr.regione.emiliaromagna.i/wcm/asr/aree_di_programma/rischioinfettiv o/gr_ist/pr_inf_ccm/pubblicazioni/pr_infoss/link/giio2 007_infoss.pdf

Moro, M.L., Petrosillo, N., Gandin, C. \& Bella, A. (2004) Infection control and hospital epidemiology in Italian hospitals: the official journal of the Society of Hospital Epidemiologists of America, 25(1): 36-40

Nicastri, E., Petrosillo, N., Martini, L., Larosa, M., Gesu, G.P., Ippolito, G. \& INF-NOS Study Group (2003) Prevalence of nosocomial infections in 15 Italian hospitals: first point prevalence study for the INF-NOS project. Infection, 31 (Suppl 2): 10-5.

Nichols, R.L. (2001) Preventing Surgical Site Infections: A
Surgeon's Perspective. Emerging Infectious Diseases, 7(2): 220-4.

Olsen, M.A., Chu-Ongsakul, S., Brandt, K.E., Dietz, J.R., Mayfield, J. \& Fraser V.J. (2008) Hospitalassociated costs due to surgical site infection after breast surgery. Archives of Surgery, 143(1): 53-60; discussion 61.

Owens, C.D. \& Stoessel, K. (2008) Surgical site infections: epidemiology, microbiology and prevention. Journal of Hospital Infection, 70 (Suppl 2):3-10.

Owens, W.D., Felts, J.A. \& Spitznagel, E.L. Jr. (1978) ASA physical status classifications: a study of consistency of ratings. Anesthesiology, 49(4): 239-43.

Petrosillo, N., Drapeau, C.M., Nicastri, E., Martini, L., Ippolito, G., Moro, M.L. \& ANIPIO (2008) Surgical site infections in Italian Hospitals: a prospective multicenter study. BMC Infectious Diseases, 8: 34.

Reilly, J., Allardice, G., Bruce, J., Hill, R. \& Mc Coubrey, J. (2006) Procedure-specific surgical site infection rates and post discharge surveillance in Scotland. Infections Control and Hospital Epidemiology, 27(12): 1318-23.

Storch, M., Scalzo, H., Van Lue, S. \& Jacinto, G. (2002) Physical and functional comparison of Coated VICRYL* Plus Antibacterial Suture (coated polyglactin 910 suture with triclosan) with Coated VICRYL* Suture (coated polyglactin 910 suture). Surgical Infections (Larchmt), 3 Suppl 1:S65-77.

Wang, Z.X., Jiang, C.P., Cao, Y. \& Ding, Y.T. (2013) Systematic review andmeta-analisis of triclosan-coated sutures for the prevention surgical-site infection. British Journal of Surgery, 100(4):465-73.

Williams, N., Sweetland, H., Goyal, S., Ivins, N. \& Leaper DJ. (2011) Randomizedtrial of antimicrobial-coated sutures to prevent surgical site infections after breast cancer surgery. Surgical Infections (Larchmt), 12(6):46974.

Williams, M. (2008) Infection control and prevention in perioperative practice, Journal of perioperative practice, 18(7), 274-8. 